\title{
Acceleration of Hericium erinaceum mycelial growth in submerged culture using yogurt whey as an alternative nitrogen source
}

\author{
Chikako Asada, Ryosuke Okumura, Chizuru Sasaki, Yoshitoshi Nakamura* \\ Department of Life System, Institute of Technology and Science, The University of Tokushima, Tokushima, Japan \\ Email: ${ }^{*}$ nnakamu@bio.tokushima-u.ac.jp
}

Received 3 August 2012; revised 11 September 2012; accepted 19 October 2012

\begin{abstract}
The effects of various carbon sources and their initial concentrations on mycelia production by Hericium erinaceum were investigated by determining the dry cell weight (DCW) and $\beta$-glucan content of mycelia in submerged culture. Glucose and xylose were superior carbon sources for promoting mycelial growth resulting in mycelial concentrations of $3.99 \mathrm{~g} / \mathrm{L}$ and 4.01 g/L, respectively; glucose was the best carbon source in terms of productivity $(0.44 \mathrm{~g} / \mathrm{L} / \mathrm{day})$. Experiments were also performed using yogurt whey as an alternative nitrogen source for submerged cultivation of $\boldsymbol{H}$. erinaceum mycelia, and DCW and $\beta$-glucan content were compared with those with chemical nutrient medium. When whey was used as a nitrogen source, DCW and total amount of $\beta$-glucan were 2.3- and 2.8-fold higher, respectively, than that with chemical nutrient medium. Thus, whey appears to be an alternative nitrogen source for promoting $\boldsymbol{H}$. erinaceum mycelial growth.
\end{abstract}

Keywords: Hericium erinaceum; Mycelia; Submerged Culture; Yogurt Whey

\section{INTRODUCTION}

Basidiomycetes are classified as fungi, and their edible fruiting bodies are known as mushrooms. In recent years, various biological and pharmacological activities of certain types of mushrooms have been revealed; for example, lentinan from Lentinus edodes and sonifilan from Schizophyllum commune are well known as anti-tumor polysaccharides [1,2]. Yamabushitake, or Hericium erinaceum, is an edible mushroom that belongs to the Aphyllophorales order and Hydnaceae (Hericiaceae) class. Members of the Hericium genus are widely distributed throughout Japan and China. Compounds isolated from $\mathrm{H}$. erinaceum show interesting biological ac-

"Corresponding author. tivities, including cytotoxic effects against HeLa cells, promotion of the synthesis of nerve growth factor (NGF), and inhibition of pollen germination [3]. Cultivation of basidiomycetes is complicated because the fruiting bodies require finely-tuned conditions of light intensity, temperature, and humidity, and are more complex than the fungal mycelia. Therefore, a rapid and efficient method for producing compounds from fungal mycelia is desired. On the other hand, whey is a by-product of the yogurt or cheese-making process and contains proteins, vitamins, minerals, and some sources of carbon. A large portion of whey is discarded as industrial garbage arising from food production, but because of the possibility of environmental pollution due to high levels of BOD, effective utilization of whey is anticipated [4-6]. Some researchers have reported that cheese whey can be used as an alternative carbon and/or nitrogen source [7-10]. However, few have studied the utilization of yogurt whey as an alternative substrate. Therefore, we focused on yogurt whey as an unutilized industrial biomass.

In this study, the optimal carbon source to obtain a large amount of $H$. erinaceum mycelia was investigated. Furthermore, we used yogurt whey as an alternative nitrogen source for submerged cultivation of $H$. erinaceum mycelia, and compared the mycelial yield and $\beta$-glucan content with those with chemical nutrient medium.

\section{MATERAIALS AND METHODS}

\subsection{Microorganism and Seed Culture}

Fungal mycelia of Hericium erinaceum was used in this study and cultured on Potato Dextrose Agar (Wako Pure Chemical Industries, Ltd.) plates at $25^{\circ} \mathrm{C}$.

\subsection{Culture Media and Cultivation}

To pre-culture the fungus, pieces of mycelia were inoculated into $200 \mathrm{~mL}$ Erlenmeyer flasks containing $50 \mathrm{~mL}$ of medium consisting of the following ingredients buffered 
with $0.4 \mathrm{M}$ phthalic acid (pH 5.0): glucose $30 \mathrm{~g} / \mathrm{L}$, polypeptone $6.3 \mathrm{~g} / \mathrm{L}$, yeast extract $0.2 \mathrm{~g} / \mathrm{L}, \mathrm{KH}_{2} \mathrm{PO}_{4} 1.0 \mathrm{~g} / \mathrm{L}$, $\mathrm{MgSO}_{4} \cdot 7 \mathrm{H}_{2} \mathrm{O} 0.5 \mathrm{~g} / \mathrm{L}$ (basal medium). All nutrients were purchased from Wako Pure Chemicals. The flasks were incubated in a rotary shaker (TAITEC, Bio-Shaker BR$300 \mathrm{LF}$ ) at $25^{\circ} \mathrm{C}, 100 \mathrm{rpm}$ for 7 days.

The main culture was carried out in $300 \mathrm{~mL}$ Erlenmeyer flasks containing $60 \mathrm{~mL}$ of basal medium. The pre-culture was homogenized with a polytron homogenizer (Model PT-MR 2100, Kinematica AG, Switzerland) for $20 \mathrm{~s}$ at $15000 \mathrm{rpm}$. Two percent (v/v) of the homogenized pre-cultures were inoculated into the main culture media. The flasks were then incubated at $25^{\circ} \mathrm{C}, 100 \mathrm{rpm}$. Different carbon sources (galactose, glucose, mannose, arabinose, xylose, lactose, maltose, and soluble starch) and initial glucose concentrations $(10,20,30,40,50 \mathrm{~g} / \mathrm{L})$ were tested.

The possible use of yogurt whey as an alternative nitrogen source was also examined. The yogurt whey was separated from commercially available yogurt by centrifuging a sample for $30 \mathrm{~min}$ at $3000 \mathrm{rpm}$ using a small centrifuge (H-110A, KOKUSAN Ltd.). The separated supernatant as a sample of yogurt whey in this study was sterilized by filtering it through a $0.20-\mu \mathrm{m}$ membrane filter (Nalge Nunc International, USA) and then subsequently used as mycelial growth medium. The effects of whey concentration $[100 \%, 75 \%, 50 \%, 25 \%(\mathrm{v} / \mathrm{v})]$ on mycelial growth were investigated. Cultivation in basal medium supplemented with $20 \mathrm{~g} / \mathrm{L}$ glucose was used as a positive control (P.C.), and cultivation without polypeptone and yeast extract as nitrogen sources was used as a negative control (N.C.).

\subsection{Analytical Methods}

After cultivation, mycelia were filtered from culture me dium through ADVANTEC no. 131 filter paper. The filtered mycelial pellet was washed with $300 \mathrm{~mL}$ distilled water and air dried for 2 days at $35^{\circ} \mathrm{C}$. Subsequently, it was stored in a vacuum desiccator with silica gel at room temperature. The dry cell weight (DCW) was then determined. Glucose concentration was determined by the mutarotase GOD method (Glucose C-Test; Wako Pure Chemicals, Osaka, Japan), the soluble starch concentration by the phenol-sulfuric acid method, and the concentrations of the other carbon sources were determined by HPLC (Shimadzu RID-10A, Shimadzu Co. Ltd., Kyoto, Japan) with an Aminex HPX-87H column (300 mm $\times 7.8$ $\mathrm{mm}$ ) (Bio-rad, Richmond, CA). Analysis of the amino acid composition of whey was also performed using HPLC (JLC-500/V, JEOL Ltd.). The solvent used in all mobile phases was sodium citrate buffer $(\mathrm{H}-01-\mathrm{H}-04)$ supplied by JEOL Ltd. The Ninhydrin Coloring Solution Kit-II for JEOL (Wako) was used for the reaction agent. The $\beta$-glucan content was assayed using the Mushroom and Yeast $\beta$-glucan Assay Kit (Megazyme International Ireland Ltd., Bray Business Park, Bray, Co. Wicklow, Ireland). All experiments were carried out in triplicate and individual assays were performed at least twice.

\section{RESULTS AND DISCUSSION}

\subsection{Investigation of the Effect of Carbon Source on Fungal Growth}

Carbohydrates are important nutrients for microbial growth. To determine the optimal carbon sources for $H$. erinaceum mycelia growth in submerged culture, 8 different carbon sources were individually assessed at concentrations of $20 \mathrm{~g} / \mathrm{L}$ by replacing glucose in the abovementioned basal medium; the results are shown in Figure 1. Galactose, arabinose, lactose, and soluble starch

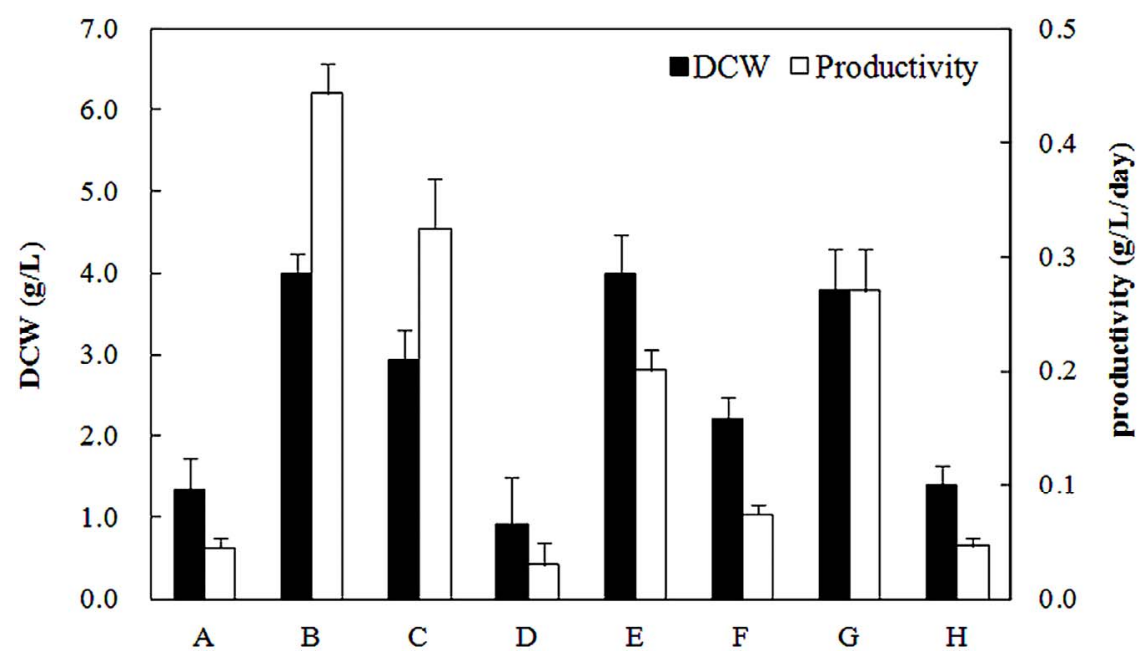

Figure 1. Effect of carbon sources on $H$. erinaceum mycelial growth incubated in basal medium supplemented with $20 \mathrm{~g} / \mathrm{L}$ of each carbon source: (A) galactose, (B) glucose, (C) mannose, (D) arabinose, (E) xylose, $(\mathrm{F})$ lactose, $(\mathrm{G})$ maltose, $(\mathrm{H})$ soluble starch. 
were not completely consumed in 30 days (data not shown). On the other hand, when glucose, xylose, and mannose were used, complete consumption was observed in 9, 20, and 14 days, respectively. The second highest DCW, $3.99 \mathrm{~g} / \mathrm{L}$, and the highest productivity, $0.44 \mathrm{~g} / \mathrm{L} /$ day, were obtained in glucose medium. Xylose yielded better mycelial growth, $4.01 \mathrm{~g} / \mathrm{L}$, than glucose. However, because it took more days to consume all of the carbon, its productivity, $0.20 \mathrm{~g} / \mathrm{L} /$ day, was less than that of glucose. Different carbon sources may result in different catabolite repression of mycelial metabolism, especially if the different carbon sources contain polysaccharides with different carbohydrate compositions [11]. Based on these results, further study of mycelial growth under different initial concentrations of carbon was performed in glucose medium.

The effects of 5 different initial glucose concentrations on $H$. erinaceum mycelial growth in submerged culture were investigated, and the results are shown in Figure 2. An initial glucose concentration of $40 \mathrm{~g} / \mathrm{L}$ gave the highest DCW, but the highest productivity was obtained at an initial glucose concentration of $20 \mathrm{~g} / \mathrm{L}$. Beyond the initial glucose concentration of $20 \mathrm{~g} / \mathrm{L}$, little increase in DCW was observed. Furthermore, the higher the initial glucose concentration, the longer it took to consume all of the glucose, and as a result the productivity was dramatically decreased. This implied that dissolved oxygen, which is critical to mycelial growth, was significantly decreased. Furthermore, the production of organic acids and secondary metabolites, such as enzymes that may inhibit mycelial growth due to end product inhibition may have occurred [1]. In particular, bacteriolysis may have occurred at an initial glucose concentration of $50 \mathrm{~g} / \mathrm{L}$ because DCW of glucose at $50 \mathrm{~g} / \mathrm{L}(3.61 \mathrm{~g} / \mathrm{L})$ was lower than that of glucose at $20 \mathrm{~g} / \mathrm{L}(3.99 \mathrm{~g} / \mathrm{L})$. As a result, it was decided that the best carbon source was glucose, and that the initial glucose concentration should be $20 \mathrm{~g} / \mathrm{L}$ to obtain a large amount of mycelia in a relatively short incubation time.

\subsection{Utilization of Whey as an Alternative Nitrogen Source}

The whey used in this study contained $1 \mathrm{~g} / \mathrm{L}$ glucose, 40 $\mathrm{g} / \mathrm{L}$ lactose, $10 \mathrm{~g} / \mathrm{L}$ galactose, $3.5 \mathrm{~g} / \mathrm{L}$ proteins, and 1.2 $\mathrm{g} / \mathrm{L}$ lactic acid; the $\mathrm{pH}$ of the whey was 4.2 . The protein content was calculated from the concentrations of the constituent amino acids as shown in Table 1. Figure 3 shows the effect of the proportion of whey in medium on $H$. erinaceum mycelial growth in yogurt whey medium. In this experiment, the glucose concentration in the medium was adjusted to $20 \mathrm{~g} / \mathrm{L}$ by addition of glucose because this glucose concentration gave the highest mycelial productivity as shown in Figure 2. Glucose was completely consumed in all of the cultures containing

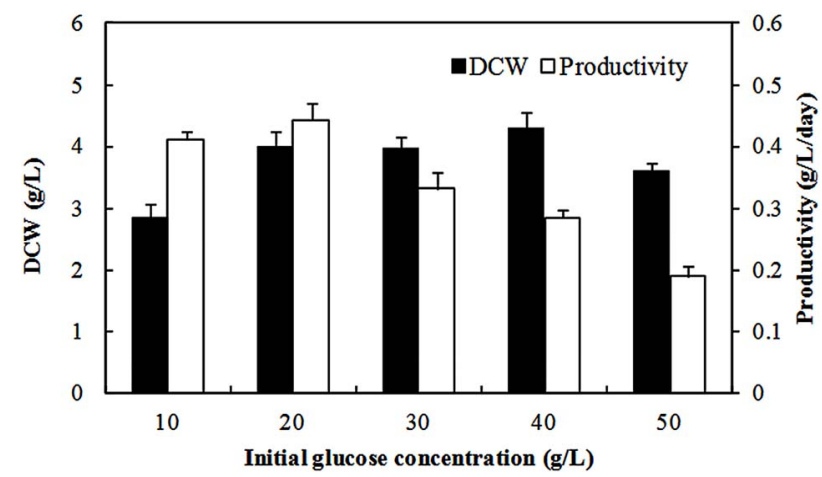

Figure 2. Effect of initial glucose concentration on $H$. erinaceum mycelial growth in basal medium.

Table 1. Concentration of amino acids in the whey used in this study.

\begin{tabular}{|c|c|c|}
\hline & $\begin{array}{l}\text { Free amino } \\
\text { acid }(\mathrm{mg} / \mathrm{L})\end{array}$ & $\begin{array}{l}\text { Constituent amino } \\
\text { acid }(\mathrm{mg} / \mathrm{L})\end{array}$ \\
\hline Alanine & 17.65 & 113.36 \\
\hline$\alpha$-amino butyric acid & 5.23 & 13.63 \\
\hline Arginine & 5.39 & 74.22 \\
\hline Asparagine & 3.61 & 652.32 \\
\hline Aspartic acid & 8.60 & 259.81 \\
\hline Cistine & 0 & 7.45 \\
\hline Citrullin & 0 & 75.76 \\
\hline$\gamma$-amino butyric acid & 70.39 & 319.94 \\
\hline Glutamic acid & 2.34 & 0 \\
\hline Glutamine & 7.66 & 0 \\
\hline Glycine & 2.31 & 45.96 \\
\hline Histidine & 6.69 & 84.12 \\
\hline Hydroxy lysine & 253.81 & 0 \\
\hline Isoleucine & 2.70 & 183.10 \\
\hline Leucine & 7.50 & 247.67 \\
\hline Lysine & 2.48 & 231.29 \\
\hline Methionine & 0 & 64.38 \\
\hline Mono ethanol amine & 48.36 & 45.07 \\
\hline Ornithine & 2.93 & 0 \\
\hline Phenylalanine & 1.69 & 124.77 \\
\hline Phospho ethanol amine & 0 & 115.79 \\
\hline Phospho serine & 38.40 & 252.49 \\
\hline Serine & 7.97 & 170.25 \\
\hline Taurine & 0 & 18.41 \\
\hline Threonine & 5.50 & 212.66 \\
\hline Tyrosine & 0 & 41.68 \\
\hline Valine & 8.12 & 217.85 \\
\hline
\end{tabular}




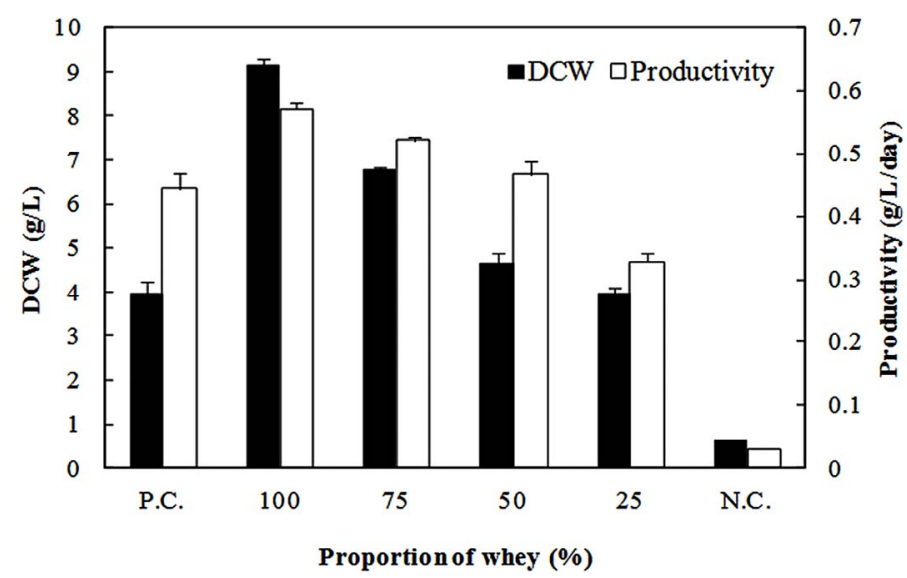

Figure 3. Effect of the proportion of whey in medium on H. erinaceum mycelial growth in yogurt whey medium. P.C. (positive control) refers to basal medium, N.C. (negative control) refers to basal medium without polypeptone and yeast extract.

Table 2. Effect of the proportion of whey in the medium on the $\beta$-glucan content of $H$. erinaceum mycelia. P.C. (positive control) refers to basal medium supplemented with $20 \mathrm{~g} / \mathrm{L}$ glucose, N.C. (negative control) refers to basal medium supplemented with $20 \mathrm{~g} / \mathrm{L}$ glucose without polypeptone and yeast extract.

\begin{tabular}{cccc}
\hline Proportion of whey $(\%)$ & Culture time (day) & $\beta$-glucan content $(\%)$ & Total amount of $\beta$-glucan $(\mathrm{g} / \mathrm{L})$ \\
\hline P.C. & 9 & $15.1 \pm 0.1$ & $0.602 \pm 0.03$ \\
100 & 16 & $18.7 \pm 0.3$ & $1.706 \pm 0.03$ \\
75 & 13 & $19.0 \pm 0.4$ & $1.285 \pm 0.01$ \\
50 & 10 & $19.4 \pm 0.0$ & $0.905 \pm 0.04$ \\
25 & 12 & $24.2 \pm 0.0$ & $0.955 \pm 0.04$ \\
N.C. & 20 & n.d. & n.d. \\
\hline
\end{tabular}

whey (data not shown). Whey rate of $50 \%(\mathrm{v} / \mathrm{v})$ was the earliest of four conditions, otherwise high whey rate made culture day longer as shown in Table 2. A high lactic acid concentration may inhibit mycelial growth. However, it was obvious that a high proportion of whey had a positive effect on mycelial yield because all the media that contained whey showed greater mycelial growth. The highest DCW was obtained with $100 \%$ whey medium, where it was about 2.3 -fold higher than that in basal medium (P.C.). In fact, it has previously been reported that mycelial biomass cultivated using casein peptone was greater than that obtained by polypeptone cultivation [12]. The $\beta$-glucan content of the mycelia cultivated in whey medium increased as the proportion of whey in the media decreased (Table 2). This trend is similar to the results found from the investigations on nitrogen concentration: The highest $\beta$-glucan content was obtained at the lowest nitrogen concentration. However, the absolute amount of $\beta$-glucan was increased when whey was used as an alternative nitrogen source. The highest absolute amount of $\beta$-glucan was obtained in $100 \%$ whey medium, and was 2.8 -fold higher than that in basal medium (P.C.). The protein content was lesser in whey medium than in polypeptone medium, but nevertheless, greater mycelial growth was observed in whey. It appears that other nutrients in whey, such as a lipid or trace elements such as vitamins, are more effective at promoting mycelial growth.

\section{CONCLUSION}

The highest $H$. erinaceum mycelial DCW and productivity $(3.99 \mathrm{~g} / \mathrm{L}$ and $0.44 \mathrm{~g} / \mathrm{L} /$ day, respectively) were obtained with an initial glucose concentration of $20 \mathrm{~g} / \mathrm{L}$ at $\mathrm{pH} 5,25^{\circ} \mathrm{C}$. Whey could be used as an alternative nitrogen source, as DCW was 2.3 -fold greater in whey medium compared with that in chemical nutrient medium, and the absolute amount of $\beta$-glucan was 2.8 -fold greater. These results indicate that yogurt whey can stimulate $H$. erinaceum mycelial growth. However, further detail studies are required to identify the effective components of yogurt whey.

\section{REFERENCES}

[1] Kurosumi, A., Kobayashi, F., Mtui, G. and Nakamura, Y. 
(2006) Development of optimal culture method of Sparassis crispa mycelia and a new extraction method of antineoplastic constituent. Biochemical Engineering Journal, 30, 109-113. doi:10.1016/j.bej.2006.02.004

[2] Malinowska, E., Krzyczkowski, W., Łapienis, G. and Herold, F. (2009) Improved simultaneous production of mycelial biomass and polysaccharides by submerged culture of Hericium erinaceum: Optimization using a central composite rotatable design (CCRD). Journal of Industrial Microbiology \& Biotechnology, 36, 1513-1527. doi:10.1007/s10295-009-0640-x

[3] Mizuno, T., Wasa, T., Ito, H., Suzuki, C. and Ukai, N. (1992) Antitumor-active polysaccharides isolated from the fruiting body of Hericium erinaceum, an edible and medicinal mushroom called yamabushitake or houtou. Bioscience Biotechnology Biochemistry, 56, 347-348. doi:10.1271/bbb.56.347

[4] Ben-Hassan, R.M. and Ghaly, A.E. (1994) Continuous propagation of Kluyveromyces fragilis in cheese whey for pollution potential reduction. Applied Biochemistry and Biotechnology, 47, 89-105. doi:10.1007/BF02788678

[5] Mawson, A.J. (1994) Bioconversions for whey utilization and waste abatement. Bioresource Technology, 47, 195203. doi:10.1016/0960-8524(94)90180-5

[6] Fournier, D., Schwitzguebel, J.P. and Peringer, P. (1993) Effect of different heterogeneous inocula in acidogenic fermentation of whey permeate. Biotechnology Letters, 15, 627-632. doi:10.1007/BF00138553

[7] Crisiani-Urbina, E., Netzahuatl-Muñoz, A.R., ManriquezRojas, F.J., Juárez-Ramírez, C., Ruiz-Ordaz, N. and Ga-
líndez-Mayer, J. (2000) Batch and fed-batch cultures for the treatment of whey with mixed yeast cultures. Process Biochemistry, 35, 649-657. doi:10.1016/S0032-9592(99)00116-8

[8] Kassa, A., Brownbridge, M., Parker, B.L., Skinner, M., Gouli, V., Gouli, S., Guo, M., Lee, F. and Hata, T. (2008) Whey for mass production of Beauveria bassiana and Metarhizium anisopliae. Mycological Research, 112, 583591. doi:10.1016/i.mycres.2007.12.004

[9] Lee, H., Song, M. and Hwang, S. (2003) Optimizing bioconversion of deproteinated cheese whey to mycelia of Ganoderma lucidum. Process Biochemistry, 38, 16851693. doi:10.1016/S0032-9592(02)00259-5

[10] Lee, H., Song, M., Yu, Y. and Hwang, S. (2003) Production of Ganoderma lucidum mycelia using cheese whey as an alternative substrate: response surface analysis and biokinetics. Biochemical Engineering Journal, 15, 93-99. doi:10.1016/S1369-703X(02)00211-5

[11] Kim, S.W., Hwang, H.J., Xu, C.P., Sung, J.M., Choi, J. W. and Yun, J.W. (2003) Optimization of submerged culture process for the production of mycelial biomass and exo-polysaccharides by Cordyceps militaris C738. Journal of Applied Microbiology, 94, 120-126. doi:10.1046/j.1365-2672.2003.01754.x

[12] Kim, H.O., Lim, J.M., Joo, J.H., Kim, S.W., Hwang, H.J., Choi, J.W. and Yun, J.W. (2005) Optimization of submerged culture condition for the production of mycelial biomass and exopolysaccharides by Agrocybe cylindracea. Bioresource Technology, 96, 1175-1182. doi:10.1016/j.biortech.2004.09.021 